\title{
Integration of Droplet Microfluidic Tools for Single-cell Functional Metagenomics: An Engineering Head Start
}

\author{
David Conchouso ${ }^{1,2,3}$, Amani Al-Ma'abadi ${ }^{2,3,}$, Hayedeh Behzad $^{2,3}$, Mohammed \\ Alarawi $^{2,3}$, Masahito Hosokawa ${ }^{4,5,6}$, Yohei Nishikawa ${ }^{4,7}$, Haruko Takeyama ${ }^{4,5,6,7}$, \\ Katsuhiko Mineta ${ }^{2,8, *}$, Takashi Gojobori ${ }^{2,3, *}$ \\ ${ }^{1}$ Department of Industrial and Mechanical Engineering, Universidad de las Américas Puebla, Puebla 72810, Mexico \\ ${ }^{2}$ Computational Bioscience Research Center, King Abdullah University of Science and Technology, Thuwal 23955-6900, Saudi Arabia \\ ${ }^{3}$ Biological and Environmental Sciences and Engineering Division, King Abdullah University of Science and Technology, Thuwal 23955- \\ 6900, Saudi Arabia \\ ${ }^{4}$ Research Organization for Nano \& Life Innovation, Waseda University, Tokyo 162-0041, Japan \\ ${ }^{5}$ Department of Life Science and Medical Bioscience, Waseda University, Tokyo 162-8480, Japan \\ ${ }^{6}$ Institute for Advanced Research of Biosystem Dynamics, Waseda Research Institute for Science and Engineering, Waseda University, \\ Tokyo 169-8555, Japan \\ ${ }^{7}$ Computational Bio Big-Data Open Innovation Laboratory, AIST-Waseda University, Tokyo 169-0072, Japan \\ ${ }^{8}$ Computer, Electrical, and Mathematical Sciences and Engineering Division, King Abdullah University of Science and Technology, \\ Thuwal 23955-6900, Saudi Arabia
}

Received 24 August 2020; revised 9 February 2021; accepted 11 June 2021

Available online 21 December 2021

Handled by Jun Yu

\begin{abstract}
Droplet microfluidic techniques have shown promising outcome to study single cells at high throughput. However, their adoption in laboratories studying "-omics" sciences is still irrelevant due to the complex and multidisciplinary nature of the field. To facilitate their use, here we provide engineering details and organized protocols for integrating three droplet-based microfluidic technologies into the metagenomic pipeline to enable functional screening of bioproducts at high throughput. First, a device encapsulating single cells in droplets at a rate of $\sim 250 \mathrm{~Hz}$ is described considering droplet size and cell growth. Then, we expand on previously reported fluorescence-activated droplet sorting systems to integrate the use of 4 independent fluorescence-exciting lasers (i.e., 405, 488, 561, and $637 \mathrm{~nm}$ ) in a single platform to make it compatible with different fluorescence-emitting biosensors. For this sorter, both hardware and software are provided and optimized for effortlessly sorting droplets at $60 \mathrm{~Hz}$. Then, a passive droplet merger is also integrated into our pipeline to enable adding new reagents to already-made droplets at a rate of $200 \mathrm{~Hz}$. Finally, we provide an optimized recipe for manufacturing these chips using silicon dry-etching tools. Because of the overall integration and the technical details presented here, our approach allows biologists to quickly use microfluidic technologies and achieve both single-cell resolution and high-throughput capability ( $>50,000$ cells/day) for mining and
\end{abstract}

*Corresponding authors.

E-mail: katsuhiko.mineta@kaust.edu.sa (Mineta K), takashi.gojobori@kaust.edu.sa (Gojobori T).

${ }^{\S}$ Current address:

Life Science and Environment Research Institute, King Abdulaziz City for Science and Technology, Riyadh 11442, Saudi Arabia.

Peer review under responsibility of Beijing Institute of Genomics, Chinese Academy of Sciences / China National Center for Bioinformation and Genetics Society of China. https://doi.org/10.1016/j.gpb.2021.12.018

1672-0229 (C) 2021 The Authors. Published by Elsevier B.V. and Science Press on behalf of Beijing Institute of Genomics, Chinese Academy of Sciences / China National Center for Bioinformation and Genetics Society of China.

This is an open access article under the CC BY license (http://creativecommons.org/licenses/by/4.0/). 
bioprospecting metagenomic data.

KEYWORDS Droplet microfluidics; Single cell; Metagenomics; Droplet sorter; Biotechnology

\section{Introduction}

Droplet microfluidic technologies are revolutionary tools enabling chemical and biological discoveries [1-5]. In biology, droplet microfluidics has an extraordinary impact on developing novel assays performed at the granularity of a single cell and high throughput [6-12]. For example, it is now possible to carry out millions of single-cell studies in microfluidic droplets acting as microreactors of only $\sim 1-1000 \mathrm{pl}$ each [13]. In these droplets, one can encapsulate single cells [6,11], insert controlled amounts of reagents $[7,12-15]$, add unique barcodes to each droplet $[7,8,16,17]$, and deliver biosensors to detect cell characteristics at unprecedented precision and high-throughput capability $[6,7,11,18]$.

Other characteristics that are key for the integration of these technologies into biological research include accurate detection of biological products $[5,11,15,18-20]$, rapid heat transfer and cycling [21], low-cost [22,23], as well as simple integration with automation, data processing tools $[6,8,18,24]$, and portable electronic devices [25].

These features create new opportunities for developing ambitious protocols to mine and discover novel genes [11] and entire genomes of environmental microorganisms $[26,27]$. The potential of droplet microfluidics is massive, as the pool of environmental microbes contains trillions of unique life forms $[28,29]$, each containing fundamental pieces of information in their genes that are the key to understanding the mechanisms of evolution and discovering new biochemical products. These protocols can also study cell-to-cell heterogeneity between cells belonging to the same microbial population $[27,30]$ or the same tissue or organ [8]. Consequently, droplet microfluidics can open the doors to numerous unexplored areas.

Traditional methods cannot practically handle single-cell studies, as $99 \%$ of the microorganisms cannot be cultured in the laboratory [29] and their throughput is low compared to the number of single-cell experiments needed. Some techniques, such as $16 \mathrm{~S}$ rRNA gene sequencing, can obtain phylogenetic relations of uncultured microorganisms [31] but cannot mine genes for bioprospecting. Other metagenomic techniques enable gene mining from environmental microbiomes by cloning exogenous DNA in surrogate host cells to perform sequence-based and function-driven analyses in a well-controlled host system [29]. Nevertheless, these methods still require many manual operations, making them impractical.

Droplet microfluidics is the only technology capable of providing the high-throughput analysis required from sample collection to sequencing. Moreover, this technology also offers seamless integration with automation [20,32,33] and digital processing tools that are vital for analyzing the Big Data produced by the gigantic pool of living microorganisms and next-generation sequencing (NGS) techniques [29,34,35].

Despite the evidence of successful microfluidic protocols for studying single cells at unprecedented throughput and resolution $[6-8,11,12]$, only a few laboratories working on "Lab on a Chip" use them. Some of the reasons behind this poor implementation are: 1) the limited access of biologists to cleanroom facilities and the equipment needed to make microfluidic chips and conduct experiments; 2) the complex and multidisciplinary nature of microfluidics (e.g., microbiology, biosensing, NGS, bioinformatics, fluid mechanics, electronics, controls, and nanofabrication); 3) the lack of standardized protocols and methodologies; and 4) the significant budget required to start a single-cell microfluidics facility. This study will focus on solving points 2) and 3) by providing critical information and engineering insights to facilitate biologists using droplet microfluidics.

Our group studies the metagenomic diversity of the Red Sea because of its unique conditions such as high salinity and high temperature [28,30,34-37]. During this process, we assembled the set of droplet microfluidic tools, here described, to perform single-cell studies directly from metagenomic libraries of the Red Sea. Including the automatic screening of these libraries based on phenotypic responses (i.e., functional metagenomics), we selectively carried out NGS and identified new species and biological products. This study presents the integration of these tools and provides technical engineering details to implement these tools in biological laboratories. The chosen methods include the main functions needed for single-cell metagenomic studies (Figure 1): 1) single-cell encapsulation in microfluidic droplets using droplet generators with two aqueous inlets (Figure 1A) or one aqueous inlet (Figure 1B) $[6,11], 2)$ fluorescence-based droplet sorting (Figure 1C) [6], and 3) passive droplet merging, which enables the addition of precise amounts of reagents to the droplets (Figure 1D) [12].

First, a guide for manufacturing these devices is provided. This guide includes recipes for producing longlasting molds on silicon using dry etching techniques. Then, single-cell encapsulation is discussed to identify critical problems arising from this process, and a guidance is provided to solve them. Later, we present technical details for building a fully automated droplet sorter using fluorescence signals, electronic controls, lasers, and optics. We also 
A Droplet generation with two inlets

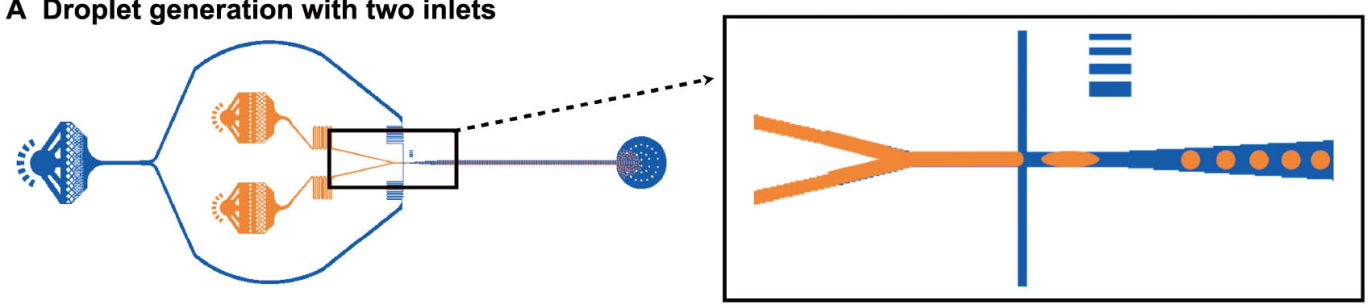

B Droplet generation with one inlet

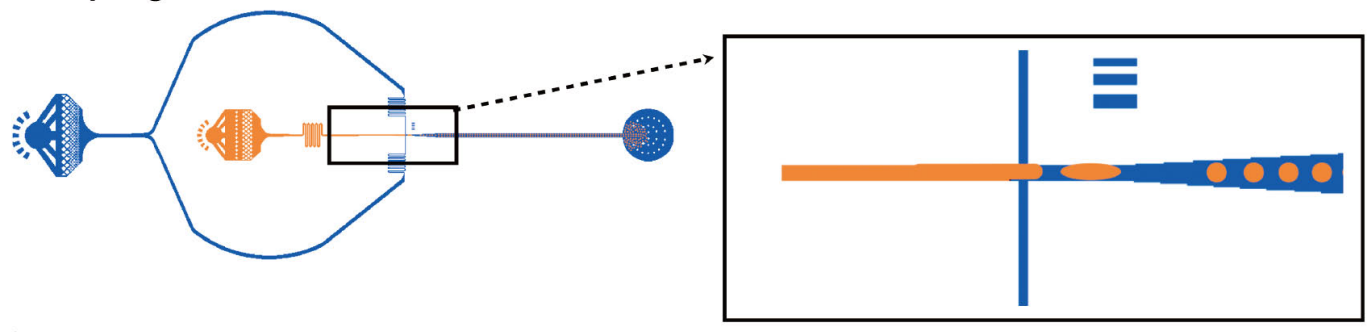

C Droplet sorter

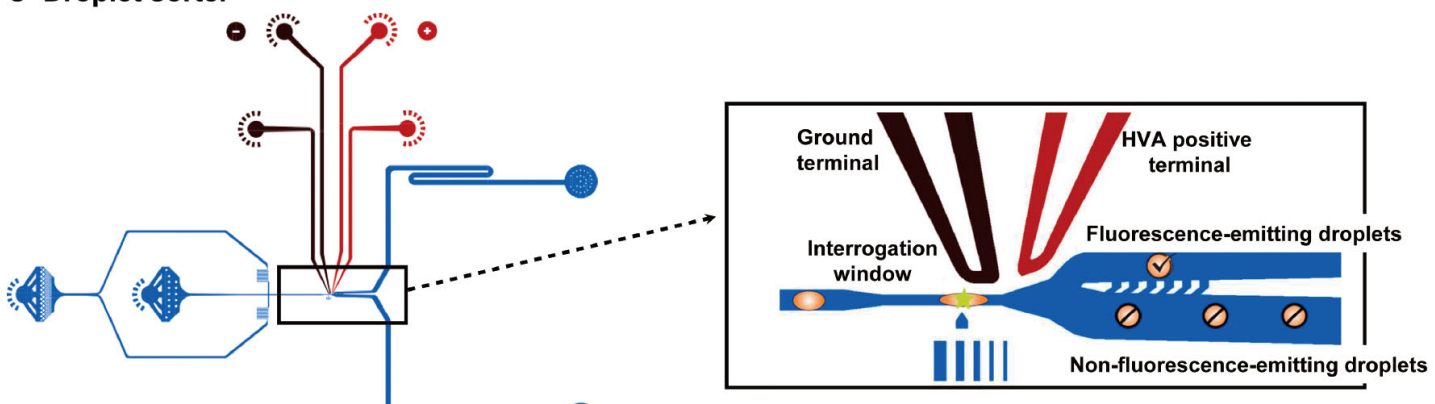

D Droplet merger

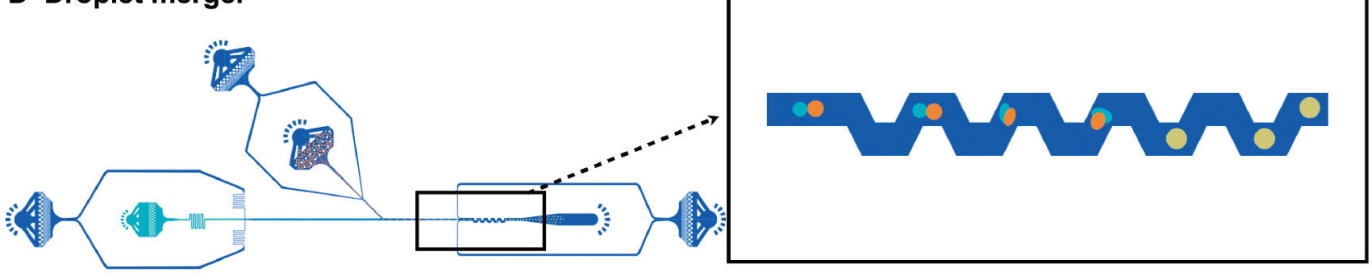

Figure 1 Droplet microfluidic techniques reviewed and integrated in this study

A. Droplet generation device for single-cell encapsulation with two inlets for introducing two different sample fluids. B. Droplet generation chip with only one inlet for the sample fluid. Both droplet generators can produce droplets at rates of 250-350 droplets/s and effectively encapsulate single cells at concentration of 0.1 cells/droplet. C. Automatic droplet sorting chip based on fluorescence. The high voltage signal applied to the terminals creates a dielectrophoretic effect, resulting in droplet sorting. The electrodes conducting the signal are colored in red and black. This picture also shows the interrogation window, where fluorescence inspection is taking place by shining each droplet with a laser and capturing the emitted light with a PMT sensor. D. Passive droplet merging device capable of merging droplets at 200 droplets/s. Note. All the channels in the insets colored in blue are filled with the oil phase, whereas channels colored in orange carry the sample fluids' aqueous phases. The designs of these devices were adapted from [6,12]. Panels A-C were adapted by permission from Mazutis and colleagues [6]. PMT, photomultiplier tube; HVA, high voltage amplifier.

provide the program developed for this procedure, insights about using the electronic instruments, and a summary of the suggested lenses and mirrors for different fluorescenceemitting biosensors working at distinctive excitation and emission wavelengths. Additionally, a passive droplet merger is described, and details of its operation are examined. Finally, we provide a discussion on the overall integration of these methods toward functional metagenomic studies.

This study is an engineering head start for any laboratory willing to incorporate droplet microfluidics into their re- search, because it gives a step-by-step guide to implement and integrate droplet microfluidic techniques into the metagenomic pipeline.

\section{Method}

Materials, equipment, and consumables used in this study

Table 1 shows a list of the materials and equipment used in this study, including reagents, optical components, device 
Table 1 Materials and equipment used in this study

\begin{tabular}{|c|c|c|c|c|}
\hline & Catalog No. & Vendor & Location & Comment \\
\hline \multicolumn{5}{|c|}{ Reagents for droplet generation } \\
\hline Pico-Wave 7500 & CO96 & Sphere Fluidics Limited & Cambridge, UK & Used as carrier fluid \\
\hline $\begin{array}{l}\text { Pico-Surf1 } 2 \%-5 \%(\mathrm{w} / \mathrm{w}) \\
\text { in NOVEC } 7500\end{array}$ & $\mathrm{CO} 24$ & Sphere Fluidics Limited & Cambridge, UK & $\begin{array}{l}\text { Surfactant for stabilizing dro- } \\
\text { plet formation }\end{array}$ \\
\hline $\begin{array}{l}\text { Trichloro }(1 \mathrm{H}, 1 \mathrm{H}, 2 \mathrm{H}, \\
\text { 2H-perfluorooctyl) silane }\end{array}$ & 448931 & $\begin{array}{l}\text { Sigma Aldrich, } \\
\text { Merck KGaA }\end{array}$ & Darmstadt, Germany & $\begin{array}{l}\text { Solution for treating master } \\
\text { molds before casting }\end{array}$ \\
\hline LB medium & L3022 & $\begin{array}{l}\text { Sigma Aldrich, } \\
\text { Merck KGaA }\end{array}$ & Darmstadt, Germany & $\begin{array}{l}\text { Aqueous media that can be } \\
\text { used as disperse phase carrier } \\
\text { for cell encapsulation }\end{array}$ \\
\hline
\end{tabular}

\section{Optical components}

$\begin{array}{lll}\text { ZT405rdc, ZT488rdc, } & \text { Chroma } & \text { BT561rdc, ZT640rdc }\end{array} \quad \begin{aligned} & \text { Bellows Falls, VT, USA } \\ & \text { Since four lasers can be used, } \\ & \text { the mirrors must be chosen to } \\ & \text { guide the four lasers' lights } \\ & \text { through the same path to the } \\ & \text { microfluidic device }\end{aligned}$

Filters

ET510/20m, ET665lp Chroma

Laser Quad Band Set for TIRF applications

TRF89901-EMv2-ET-405/ Chroma $488 / 561 / 640 \mathrm{~nm}$

Bellows Falls, VT, USA

Filters are needed to enable only a narrow bandwidth to pass to the PMT and the highspeed camera

Bellows Falls, VT, USA This cube is needed to guide the laser lights from the epifluorescent port to the excitation area and then to the output ports

Optical mounts and stands

Thorlabs

Newton, NJ, USA

PMT

H10722-20

Hamamatsu Photonics

K. K.

Hamamatsu City, Japan

These are required to mount all optical components

The PMT produces a voltage output $( \pm 5 \mathrm{~V})$ that is proportional to the intensity of the received light; this photosensor detects light in a wide wavelength range (400-800 nm)

\section{Sapphire LP/LPX laser of} $488 \mathrm{~nm}$

Sapphire 488 LP

Coherent

Santa Clara, CA, USA

These CW lasers are used to provide high-performance excitation sources for various types of fluorescent biosensors

OBIS LX/LS lasers of 405 , 561 , and $637 \mathrm{~nm}$

OBIS 405LX, OBIS 561LS, Coherent OBIS 637LX

Santa Clara, CA, USA

These CW lasers are used to provide high-performance excitation sources for various types of fluorescent biosensors

High-speed camera

FastCAM SA-Z type 200K FastCAM

Nikon

Chicago, IL, USA

Eclipse Ti-U

Nik

Tokyo, Japan

Inverted microscope with two output ports to connect the PMT and the high-speed camera

\section{Materials for fabrication}

P-type $\{100\}$ silicon wafer $400 \mu \mathrm{m}$, single side polished

University Wafer

\section{PDMS Sylgard 184}

761036

Aquapel water repellent

47100

solution

AZ ECI 3027

Photoresist

Sigma Aldrich, Merck

KGaA

PGW Auto Glass

Plasma cleaner

DRIE with ICP
PlasmaPro 100 Estrelas

\section{PDC-002 (230V)}

\section{Microchemicals GmbH}

Harrick Plasma

Oxford Instruments
Other high-speed cameras with framerates $\sim 2 \mathrm{kHz}$ can also work

It allows to observe the droplet production, excite and detect fluorescent biosensors, and record all processes 


\begin{tabular}{llll}
\hline$($ Continued $)$ & Catalog No. & Vendor & Location \\
\hline FPGA & NI-7852R & National Instruments & Austin, TX, USA \\
& & & \\
DAQ & NI SCB-68A & National Instruments & Austin, TX, USA \\
HVA & & & \\
& $623 \mathrm{~B}$ & Trek & Lockport, NY, USA
\end{tabular}

Comment

The FPGA board is the device taking the decisions based on the logical inputs; the analog input configuration is set to the differential configuration

This DAQ unit is used as the interface between the PMT and the FPGA; it is configured in direct feedthrough mode Trek HVA amplifies the train of pulses from the waveform generator; 600-800 $\mathrm{V}$ is required to drive the sorter; another critical parameter is the bandwidth of DC (e.g., $>40 \mathrm{kHz}$ ) that enables to follow the waveform signal

\begin{tabular}{|c|c|c|c|c|}
\hline Waveform generator & $33500 \mathrm{~B}$ & Keysight & Santa Rosa, CA, USA & $\begin{array}{l}\text { This device is triggered using } \\
\text { the FPGA and DAQ to send a } \\
\text { train of pulses }\end{array}$ \\
\hline $\begin{array}{l}\text { Oscilloscope InfiniiVision } \\
2000 \text { X-Series }\end{array}$ & DSO-X 2014A & Keysight & Santa Rosa, CA, USA & $\begin{array}{l}\text { Instrument used for visualiz- } \\
\text { ing the electrical signals }\end{array}$ \\
\hline \multicolumn{5}{|c|}{ Microfluidic and fluidic handling equipment } \\
\hline $\begin{array}{l}\text { The MFCS flow rate } \\
\text { platform provided with } \\
\text { four } 0-2000 \text { mbar pressure } \\
\text { sources, a flow board, and } \\
\text { four flow sensorssize S }\end{array}$ & $\begin{array}{l}\text { MFCS-EZ, Flowboard } \\
\text { FLU-S-D }\end{array}$ & Fluigent & Lowell, MA, USA & $\begin{array}{l}\text { Pressure-based platform for } \\
\text { controlling and measuring the } \\
\text { flow rates of the oil and } \\
\text { aqueous solutions through the } \\
\text { channels }\end{array}$ \\
\hline
\end{tabular}

Note: LB, lysogeny broth; TIRF, total internal reflection fluorescence; PMT, photomultiplier tube; CW, continuous-wave; PDMS, polydimethylsiloxane; DRIE, deep reactive ion etching; ICP, inductively coupled plasma; FPGA, field programable gate array; DAQ, data acquisition; HVA, high voltage amplifier; DC, direct current.

fabrication materials, and microfluidic and electronic equipment. We also report on model numbers, names, and locations of the vendors to quickly obtain the exact products. A picture of the facility designed to integrate the three microfluidic technologies, here described, is shown in Figure S1.

The MFCS series pressure controllers from Fluigent are used to drive the fluids through the microfluidic chips. These controllers have a pressure range from 0 to $700 \mathrm{kPa}$ and were managed through the company's proprietary software MAESFLO. The flow rates were measured using flow-rate sensors from the same company. Although these sensors are usually calibrated for water, it is possible to calibrate them to work with other fluids using a proportionality parameter.

\section{Fabrication method for manufacturing silicon master molds for soft lithography}

Various manufacturing techniques for microfluidic devices were developed using techniques borrowed from the microelectro-mechanical system (MEMS) industry. Examples include lasers and conventional milling machines [32,33,38,39], hot embossing [40], injection molding [41], digital light-projecting [42], 3D printing [43,44], capillary assembly [45], poly(ethylene)-glycol-diacrylate (PEGDA) casting [46], and paper-based patterning amongst others.

Soft lithography $[6,47,48]$ is the most popular technique for fabricating microfluidic chips. This process involves creating high-quality master molds for casting polydimethylsiloxane (PDMS) replicas. Although soft lithography was initially designed as a rapid prototyping technique for testing microfluidic designs, several protocols and commercially available products consider using PDMS chips in single-cell studies because of the following advantages: PDMS devices are affordable, the replicas inherit the high resolution of master molds, PDMS is biocompatible, and there are several available surface-crafting techniques to render the wetting properties [49-52].

A disadvantage of using PDMS is the low reusability of the devices in biological applications. One cannot use the same chip twice due to cross-contamination between experiments. Therefore, many PDMS chips are required daily, and consequently, the master molds must last for numerous casting processes. In this situation, the master mold's lifetime is critical. Here, we describe the most common challenges associated with the fabrication of these molds by two methods, SU-8 lithography and bulk micromachining in silicon.

\section{Fabrication challenges and benefits of SU-8 master molds}

SU-8 lithography is an excellent technique for making master molds for soft lithography, because SU-8 photoresist can be deposited at different thicknesses (e.g., 0.5-200 $\mu \mathrm{m})$ and can also be patterned at high-aspect ratios (usually $>10: 1$ ). This method also allows for creating multi-height structures with 
a smooth surface, making it a versatile technology for creating high-quality masters $[6,53]$.

Although SU-8 presents excellent advantages for rapid prototyping and low-cost manufacturing of masters, depositing thick SU-8 layers ( $>50 \mu \mathrm{m})$ can become challenging for someone unfamiliar with photolithography due to the high viscosity of the photoresist. An inexperienced person may face problems with thick SU-8 layers, such as trapped bubbles, nonuniform spin-coating, poor adhesion to the wafer, incorrect UV exposure times, internal stresses $[54,55]$, and poor mask contact resulting in low-feature resolution due to diffraction [56].

SU-8 masters can be used for many PDMS casting procedures, demonstrating excellent lifetime usage. However, sometimes these molds can show signs of wear, and the structures can be peeled off after some casting cycles due to any of the fabrication challenges mentioned above.

\section{Fabrication of silicon-based master molds using dry etching tools}

Another type of master molds, with a long-lasting lifetime, is made by bulk micromachining directly on a silicon wafer using deep reactive ion etching (DRIE) and cleanroom facilities $[57,58]$. Although this process requires specialized equipment and not all the laboratories have access to it, the fabrication of this type of master molds becomes less variable and more robust than SU-8.

These molds can be used indefinitely to make PDMS chips, showing exceptional lifetime usage as features are made in bulk silicon. The DRIE process is not trivial, but we provide a recipe for Oxford Instruments' DRIE tool with inductively coupled plasma (ICP) as a starting point for anybody who wants to manufacture these devices for the first time.

The process begins with a new p-type $\{100\}$ silicon wafer, in which an ECI-3027 photoresist mask is deposited and patterned using standard lithography. We selectively etch the silicon and create high-aspect-ratio features directly onto the silicon substrate (Figure 2). The process details are described below.

\section{Mask printing}

This process was performed using a $\mu$ PG-101 from Heidelberg Instruments. The mask designs were modified from previous studies $[6,12]$, and they were printed in their light field configuration for positive photoresist (i.e., opposite to SU-8 masking). A copy of the mask files for photolithography is provided as Datasets S1-S3.

\section{Wafer pretreatment (optional)}

The wafer was pretreated to improve the adhesion of the photoresist by removing the existing natural oxide layer. This treatment was achieved by submerging the wafer in a 20-s buffered oxide etch (BOE) bath, rinsing with deionized (DI) water for $10 \mathrm{~min}$, and drying on a hot plate. Warning, BOE contains hydrofluoric (HF) acid, and improper handling may result in serious injuries or death. A second possible pretreatment is to deposit a thin film of the hexamethyldisilizane (HMDS) adhesion promoter; however, this process requires specialized equipment [59].

\section{Standard photolithography}

A 4- $\mu \mathrm{m}$ layer of ECI-3027 positive photoresist was spun, prebaked, exposed, and developed to act as a mask to etch

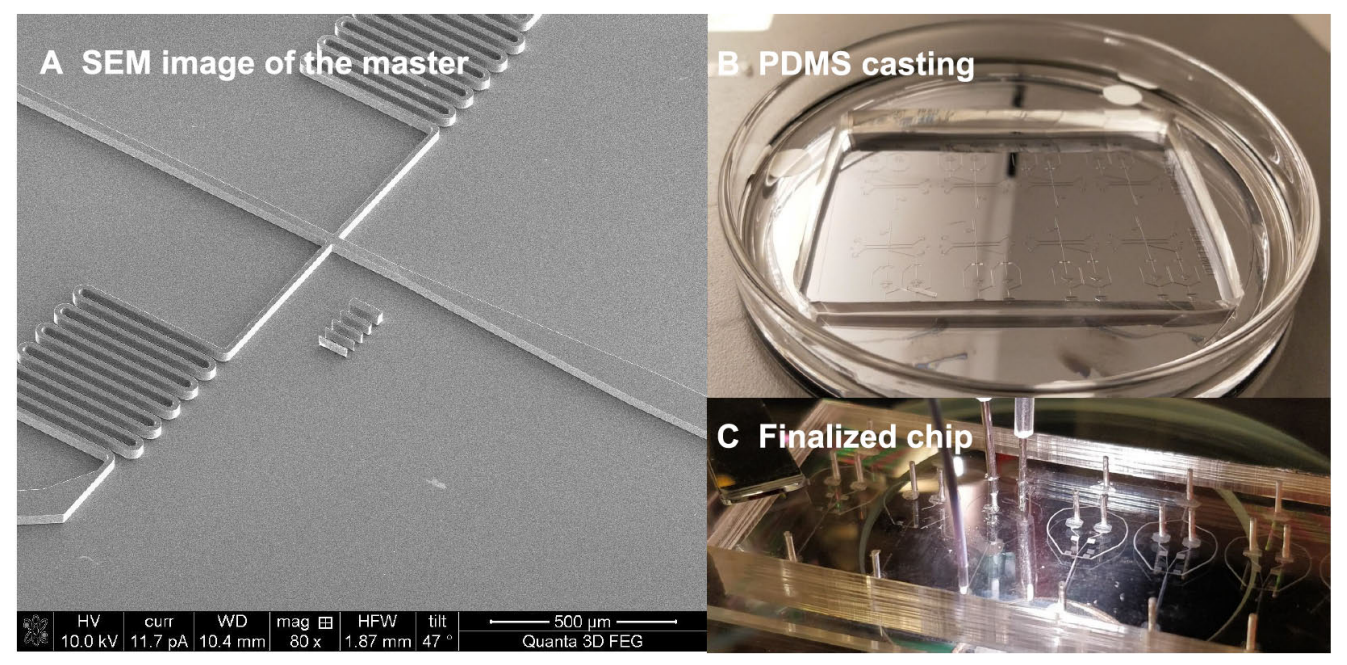

Figure 2 Fabrication of silicon-based master molds and PDMS chips

A. SEM image of the master mold for a droplet generator device. The channel features are well defined with smooth surfaces and straight walls. B. Casting of PDMS over the master mold. We prepared a mixture of the polymeric base and the curing agent (10:1) as suggested by the Sylgard® 184 Silicone-based Elastomeric Kit. This mixture was degassed in a vacuum chamber and then poured on top of the silicon master. Once the PDMS mixture is fully crosslinked, the inlets/outlets are punched, and the resulting PDMS replica is bonded to a glass slide using $\mathrm{O}_{2}$ plasma treatment. C. The inset presents an example of a finalized chip. SEM, scanning electron microscopy; PDMS, polydimethylsiloxane. 
Table 2 Process parameters for DRIE etching the master molds on silicon

\begin{tabular}{lllllll}
\hline Cycle step & $\begin{array}{l}\text { C4F8 gas flow } \\
(\mathbf{s c c m})\end{array}$ & $\begin{array}{l}\text { SF6 gas flow } \\
(\mathbf{s c c m})\end{array}$ & $\begin{array}{l}\text { RF generator power } \\
\text { (W) }\end{array}$ & $\begin{array}{l}\text { ICP generator power } \\
(\mathbf{W})\end{array}$ & $\begin{array}{l}\text { Step time } \\
\text { (s) }\end{array}$ & $\begin{array}{l}\text { Chamber pressure } \\
(\mathbf{m} \text { Torr) }\end{array}$ \\
\hline Etching & 5 & 120 & 30 & 1300 & 7 \\
Passivation & 100 & 10 & 5 & 1300 & 35 \\
\hline
\end{tabular}

Note: This recipe has been optimized for manufacturing the master molds for the devices here presented. The DRIE process requires both etching and a passivation cycle to create high aspect ratio structures. RF, radio frequency.

the silicon wafer selectively. Since this is a standard procedure, the resulting lithography was uniform and welldefined.

\section{DRIE}

This process consists of a series of etching and deposition ( $i$. $e$, passivation) cycles that alternate continuously to produce high-aspect structures on silicon. A summary of the process parameters is shown in Table 2. The initial conditions for the process were set to achieve a high vacuum (e.g., $7.5 \times 10^{-9}$ Torr) and reach a stable temperature of $-20{ }^{\circ} \mathrm{C}$. The number of cycle repetitions depends on the feature density in our wafer. If the feature density is low, there is a larger surface area to etch away, and therefore, the etching rate is slow (e.g., $\sim 0.328 \mu \mathrm{m} /$ cycle for the droplet generator chip). Conversely, if the feature density is high, there is less silicon to remove, and the etching rate is fast ( $\sim 0.4 \mu \mathrm{m} /$ cycle for the droplet sorter chip). In any case, a feature with a height of $50 \mu \mathrm{m}$ takes from $\sim 120-150$ cycles. Figure 2A shows a scanning electron microscopy (SEM) image of the silicon mold.

\section{Photoresist strip}

The remaining ECI-3027 photoresist was then stripped away using acetone.

\section{Oxygen plasma clean}

In the case of any remaining photoresist left in the silicon wafer, 30-s $\mathrm{O}_{2}$ plasma clean is recommended. This process also helps to activate the surface in preparation for the next step.

\section{Pretreatment for PDMS casting}

The masters were treated with trichloro $(1 \mathrm{H}, 1 \mathrm{H}, 2 \mathrm{H}, 2 \mathrm{H}-$ perfluorooctyl) silane to facilitate demolding. A drop of 50-100 $\mu \mathrm{l}$ was poured into a container and placed next to the master in a closed chamber. A vacuum was then applied for $4 \mathrm{~h}$ to evaporate and deposit the silane on the master's substrate. Caution, silane is toxic, and this step must be performed under a fume hood.

\section{PDMS casting (Figure 2B) and bonding}

After the master was completed, one can follow the standard soft lithography procedures $[6,47,48]$.

Fabricated devices are shown in Figure 2C. To store these chips, we recommend covering all surfaces with frosted Scotch tape until their first use to avoid capturing dust and debris on the chip's surface. Finally, the channels' walls were rendered to favor the wetting properties of the carrier fluid. Since fluorocarbon (FC) oils such as FC-40 and HFE-7500 are commonly used in single-cell experiments, one must render the PDMS channels hydrophobic. A couple of methods to achieve this surface modification include flushing the devices with Aquapel water-repellent solution $(30 \mathrm{~s})[6,7]$ or with a $1 \%$ trichloro $(1 \mathrm{H}, 1 \mathrm{H}, 2 \mathrm{H}, 2 \mathrm{H}-$ perfluorooctyl) silane solution in HFE-7500 oil (v/v) $[60,61]$. In both cases, the devices must be rinsed with the oil and blow-dried immediately after treatment $[11,12,62]$. This rinsing step is of utmost importance for Aquapel treatment because this solution can quickly react with oxygen and form particles that clog the devices.

\section{Single-cell encapsulation in droplet microfluidics}

Single-cell encapsulation is the basis of this research. Millions of cells can be individually analyzed in a pico-liter ( $\mathrm{pl}$ ) droplet that acts like a micro-Eppendorf tube where only the appropriate reagents and a single cell are present. Two critical factors are relevant for encapsulating only one cell per droplet: generation of droplets with stable and uniform size and a constant cell concentration in the sample fluid $[6,11]$. Here, we describe some of the possible solutions to address these challenges.

Microfluidic droplet generation results from the interaction between two immiscible fluids forced to flow together inside a microchannel. Droplet formation then occurs when the interfacial tension and the fluid's viscous force are balanced [32]. Although there are different channel configurations for droplet generation [5], flow-focusing generators are frequently preferred for single-cell encapsulation $[6-8,11,63]$. In this configuration, the droplet size heavily depends on the flow rate ratio of two liquids. The higher the oil-water flow rate ratio is, the smaller the produced droplets [64]. The droplet's volume is proportional to the cubic of the characteristic length; therefore, one must carefully choose a droplet size and keep it constant throughout the experiments. For example, a droplet of $100 \mu \mathrm{m}$ in diameter has a volume of $524 \mathrm{pl}$, whereas a $40-\mu \mathrm{m}$ droplet has only $33.5 \mathrm{pl}$. Therefore, a slight difference in droplet size can directly affect the encapsulation efficiency.

Additionally, if droplets have different sizes, we may compromise the droplets' operational handling in the other 
two processes (i.e., droplet sorting or merging). Therefore, the size of the droplets must be carefully designed. In the case of our experiments, the droplet size was $45 \mu \mathrm{m}$ in diameter.

The stability of the droplets is addressed by modifying the surface tension between the liquids. Usually, this can be achieved using surfactants such as $2 \%-5 \%(\mathrm{w} / \mathrm{w})$ PicoSurf1 [11] (Sphere Fluidics Limited, Cambridge, UK) or 2\% (w/w) deprotonated Krytox [7] surfactant in FC oils.

Cell concentration can be measured optically using a bright-field microscope and a hemocytometer or electronically with a cell counter. The cell concentration must be 0.1 cells/droplet to avoid encapsulating more than one cell per droplet $[11,65]$. Working with living cells in nutrientrich solutions [66,67], such as E.coli-based metagenomic libraries in lysogeny broth (LB) medium, brings other complications to cell encapsulation efficiency. Cell division can occur in 20-60 min [68], and therefore, the cell concentration may not remain constant at 0.1 cells/droplet during the encapsulation process. Cell division can be delayed by lowering the temperature of the tubing in an ice bath. If cell concentration varies due to gravity, adding OptiPrep density gradient medium from Sigma-Aldrich can reduce this problem.

\section{Fluorescence-activated droplet sorting}

One of the biggest challenges of working simultaneously with millions of droplets (i.e., experiments) is to quickly screen positive droplets from negative ones. This task requires automation and electronics to properly handle the throughput needed [69-71]. Here, we describe six essential elements to sort the droplets selectively: detection signal, control system, actuation mechanism, connection diagram and flow of information, optics and fluorescent excitation using lasers, and software development [71].

\section{Detection signal}

In metagenomics, fluorescent biosensors are commonly used to detect a phenotypic response in cells. These biosensors can be chosen based on particular enzymatic functions; thus, they can help us narrow down the specific cells of interest with a high probability of obtaining novel findings. In this regard, fluorescent biosensors are vital elements that enable the functional detection of targeted droplets.

\section{Control system}

A control system is a decision-making unit enabling time synchronization between detecting a positive droplet and switching on the actuation mechanism [71]. The system's response time is a critical design parameter to achieve efficient sorting. The overall response time must consider the reaction time of the electronic instruments, and the time required by the actuation mechanism. Those two times should then be synchronized with the speed of the moving droplets.

\section{Actuation mechanism}

Several actuation mechanisms can be used to manipulate droplets in microfluidic channels. These mechanisms include magnetic field manipulation, pneumatic control, dielectrophoretic action, acoustic wave interactions, and thermally actuated mechanisms [71]. Among these techniques, dielectrophoretic action was chosen in this study, because it offers the fastest actuation time (e.g., sorting up to $30 \mathrm{kHz}$ [69]) and is the best candidate for single-cell screening at high throughput $[6,69,72]$. Dielectrophoretic sorting consists of selectively manipulating the droplets by activating a high-voltage alternating current (AC) electric field in a localized area of a microfluidic channel. This field induces a dipole moment to slightly push a positive droplet to a different channel using a "Y-junction" $[6,69,72]$.

\section{Connection diagram and flow of information}

We show the equipment and connections to successfully implement the dielectrophoretic droplet sorter in this subsection. The design of the sorter was obtained from Mazutis and colleagues [6]. However, we focus on expanding some technical details because biologists are generally unfamiliar with these electronic components. Figure 3 shows the flow of information from one component to the other. The process starts by detecting a fluorescence signal and ends when the high-voltage AC signal is triggered at the microfluidic chip, sorting the positive droplets out. The processing time for executing these electronic commands across the different instruments was measured to be $360 \mu$ s. The effective sorting rate achieved with this system was limited by the speed of the passing droplets but not by the electronic instrumentation.

\section{Optics and fluorescent excitation using lasers}

The implementation of four laser sources (i.e., 405, 488, 561 , and $637 \mathrm{~nm}$ ) for exciting different fluorescence-emitting light biosensors using the same setup is presented in Figure 4. By selecting mirrors that reflect specific wavelengths and transmit the others (Table 3), one can combine the light path of four or more lasers, as shown in Figure 4A. Alternatively, these lasers can be mounted on an OBIS LX/ LS Laser box, and then the lasers' light can be guided to an inverted microscope by optical fibers.

Once excitation light arrives at the inverted microscope, it hits a filter box and then goes to the sorting chip through the objective lens. The emitted light then bounces back across the filter box and arrives at the high-speed camera 


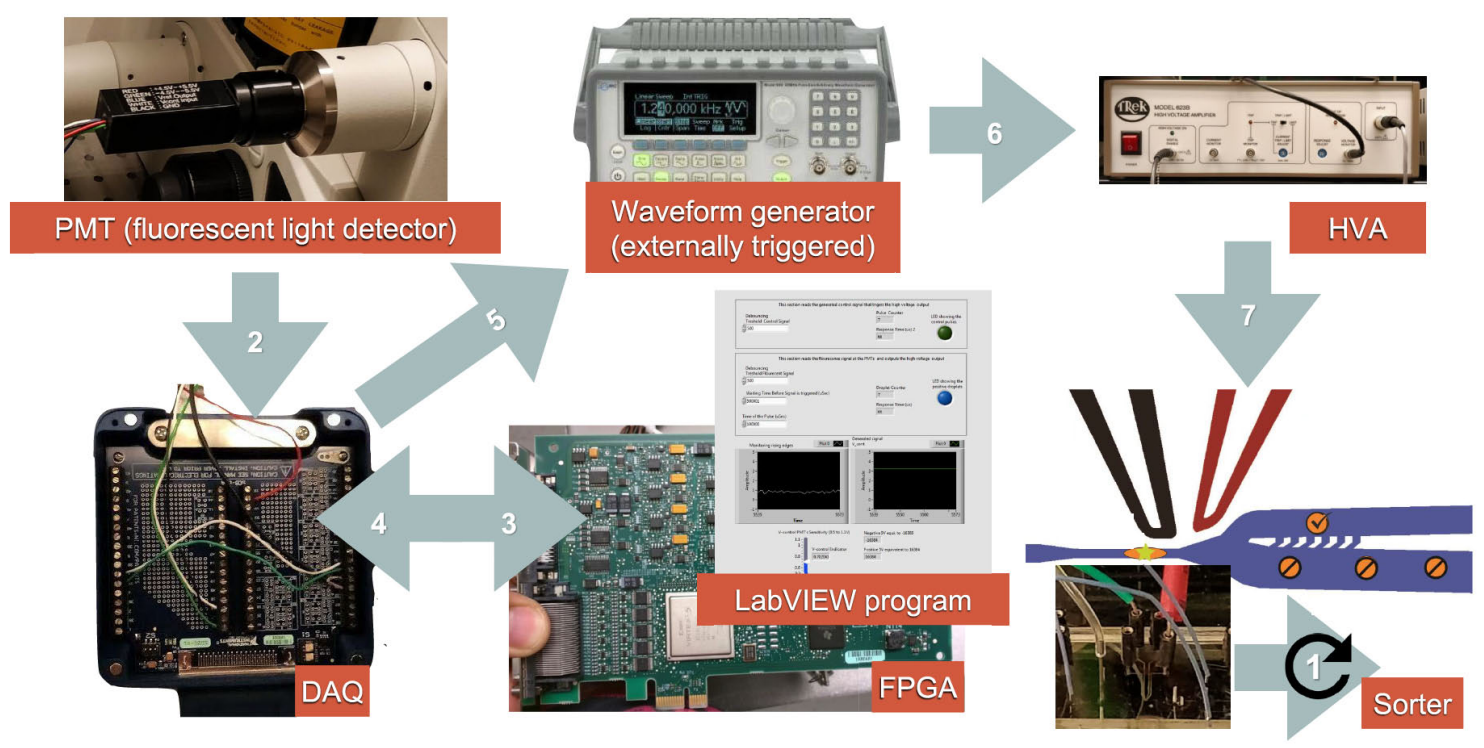

Figure 3 Flow of information through the automatic droplet sorting chip

When the PMT sensor detects fluorescent light, it sends a "true" signal to the FPGA board through the DAQ board. The FPGA logic board then decides to send back a triggering signal to the waveform generator (externally triggerred) also through the DAQ board. On this command, the waveform generator creates a train of 600 pulses at a frequency of $30 \mathrm{kHz}$, with an amplitude of $500 \mathrm{mVpp}$ and a DC offset of $250 \mathrm{mV}$. Simultaneously, the output of the waveform generator is amplified at a ratio of 1:1000 by the HVA and sent to the microfluidic chip terminals. The reaction time for the electric signals was measured to be $360 \mu \mathrm{s}$. FPGA, field programable gate array; DAQ, data acquisition; DC, direct current.

A Laser-light combiner

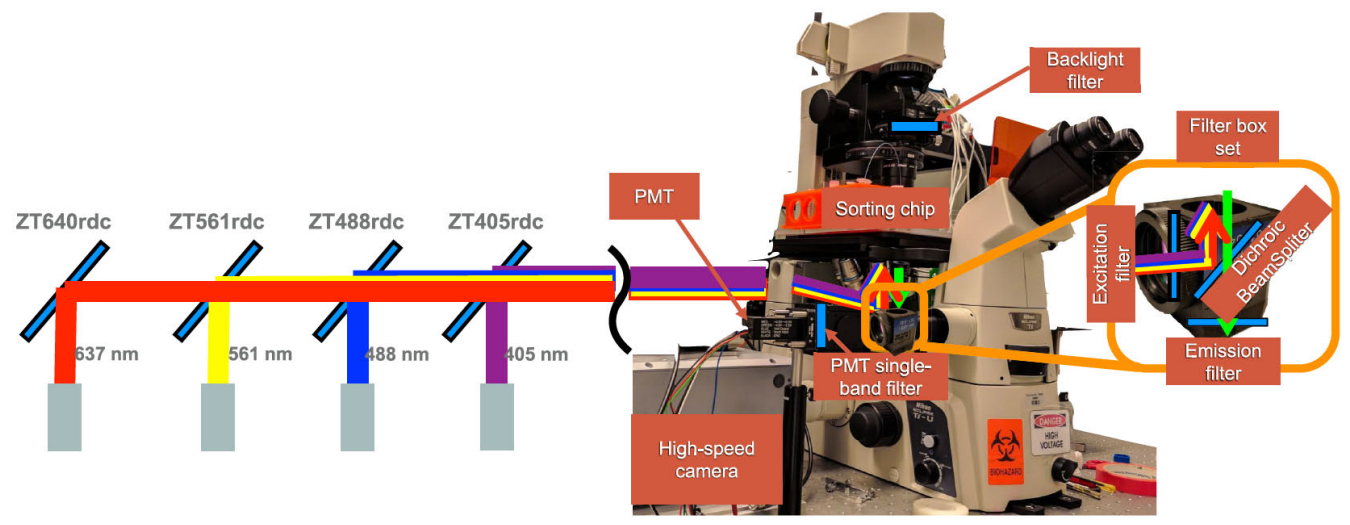

Figure 4 Lasers' path and optical setup for fluorescence detection

A. Laser-light combiner. Dichroic mirrors are used to align the laser beams in a single path guided to the epi-fluorescent illumination port of the microscope. The mirrors are selected to pass the light wavelengths to their left and reflect the wavelength of the corresponding laser. Using this configuration, one can excite the droplets with one or more laser sources. B. Optical setup and light's path through the inverted microscope. The excitation light travels from the input port to the sample chip, and the emitting light goes from the chip to the high-speed camera and the PMT ports. A key element reflecting and filtering the light is the filter box placed at the inverted microscope center.

and the photomultiplier tube (PMT) ports at a ratio of 90\%:10\% (Figure 4B). The filter box is at the center of the microscope and selectively filters and directs the excitation and emitting lights to the right locations. The filter box is a set of components composed of an excitation filter, an emission filter, and a dichroic mirror. Each of these components must be chosen depending on the excitation and emitting wavelengths of the biosensor. Fortunately, there are filter box sets that are compatible with two or more laser wavelengths. For example, the Laser Quad Band Set from Chroma (Catalog No. TRF89901-EMv2-ET-405/488/561/ $640 \mathrm{~nm}$ ) can be used with the four proposed laser wavelengths.

Connecting a high-speed camera and a PMT sensor at the same time is challenging. On the one hand, the high-speed camera requires an intense illumination backlight to record high-speed video. On the other hand, the PMT sensor is extremely sensitive, and it gets quickly saturated even with 
ambient light. For this reason, we use a beam splitter of a 90\%:10\% ratio to send most of the light to the camera port. Additionally, we installed a backlight filter and a PMT singleband filter to allow only a narrow wavelength band to pass through each sensing device. These two filters are required to have independent wavelength bands, and their selection depends on the fluorescent biosensor of interest. For example, a biosensor emitting $517 \mathrm{~nm}$ light can use an ET510/20m as a PMT filter and an ET665lp as a backlight filter.

Since the four laser sources are installed on the same platform, they can be used simultaneously or independently to excite the passing droplets. However, the sorting device has only a single "Y-junction", and therefore, it can only sort droplets in two different groups: fluorescence-emitting droplets and non-fluorescence-emitting droplets. If more than two groups are required, one must repeat the process as many times as different wavelengths exist.

\section{Software development}

The use of field programmable gate arrays (FPGAs) has enabled the design of mid-to-high-complexity systems in a programable and manageable way [73]. Since droplet sorting requires fast response times ( $\mu$ s range), we decided to implement the sorting controls on LabVIEW FPGA hardware (Figure 3 ). The code running on this device has a clock cycle of only $25 \mathrm{~ns}$, thus allowing our entire program to execute in only $66 \mu \mathrm{s}$. This program comprises three subroutines and a user interface as depicted in Figures S2-S5. Figure S2 shows the subroutine for configuring and initializing the PMT, and Figure S3 illustrates the subroutine for reading the PMT sensor and sending a triggering signal to the function generator. Likewise, Figure S4 presents a subroutine for simulating the detection of a positive droplet, and finally, Figure S5 shows the overall user interface.

The connection between the microfluidic system and the FPGA hardware was achieved using a data acquisition (DAQ) board (Figure 3). Table 4 describes all the wiring required to run the FPGA program and implement the sorting system.

The system also requires a high voltage amplifier (HVA) to produce a large electric field capable of deviating a positive droplet to a different output utilizing dielectrophoresis.

One of the most crucial factors for successfully implementing this sorting device is to measure the time it takes for a droplet to go from the laser interrogation window to the beginning of the splitting channels (Figure 1C). This time is measured with a high-speed camera, and then it is used to synchronize the controls with the droplets' flow effectively. A useful tool to evaluate droplets' speed in a channel from a high-speed video was reported by Basu and

Table 3 Optical set up

\begin{tabular}{lllll}
\hline $\begin{array}{l}\text { Laser source } \\
\text { wavelength (nm) }\end{array}$ & $\begin{array}{l}\text { Combining dichroic } \\
\text { mirror from Chroma }\end{array}$ & $\begin{array}{l}\text { Reflecting light } \\
\text { range (nm) }\end{array}$ & $\begin{array}{l}\text { Transmitting } \\
\text { light range (nm) }\end{array}$ & Example of compatible fluorochromes \\
\hline 405 & $392-415$ & $>432$ & $\begin{array}{l}\text { TagBFP, Brilliant Violet 510, Pacific Blue, Brilliant Violet } \\
570, \text { Brilliant Violet 605 }\end{array}$ \\
488 & ZT405rdc & $400-491$ & $>498$ & $\begin{array}{l}\text { DiO, LysoTracker Yellow HCK-123, EGFP, GFP, Cy2, } \\
\text { Emerald GFP, BB515, FITC, MitoTracker Green FM/MeOH, } \\
\text { Oregon Green 488, Abberior rsEGFP, DyLight 488, FAM, } \\
\text { mWasabi, Calcein, Fluo-4, Alexa Fluor 488, SYBR Green I }\end{array}$ \\
561 & ZT488rdc & & Abberior Flip 565, CAL Fluor Red 590, Rhodamine Red-X \\
637 & ZT561rdc & $730-564$ & $>652$ & $\begin{array}{l}\text { Nile Blue, Abberior Star Red, Abberior Star 635, TO-PRO-3, } \\
\text { Atto 647N, DiD, MitoTracker Deep Red 633/MeOH, Draq5, } \\
\text { Quasar 670 }\end{array}$ \\
\hline
\end{tabular}

Note: Dichroic mirrors were selected depending on the chosen laser sources. These optical components only reflect light in a defined range wavelength while allowing other wavelengths to pass through.

Table 4 Electrical connections required to run the FPGA program

\begin{tabular}{|c|c|}
\hline Signal & Connector \\
\hline \multicolumn{2}{|l|}{ Writing the PMT } \\
\hline Positive power supply $(+5 \mathrm{~V})$ & Connector0/AO3 \\
\hline Positive power supply $(-5 \mathrm{~V})$ & Connector0/AO4 \\
\hline Sensitivity $(0.5-1.1 \mathrm{~V})$ & Connector0/AO5 \\
\hline \multicolumn{2}{|l|}{ Control pulse } \\
\hline Pulse sent to the external trigger of the waveform generator & Connector0/AO1 \\
\hline \multicolumn{2}{|l|}{ Reading the PMT } \\
\hline Input port used to graph the PMT signal & Connector0/AI1 \\
\hline \multicolumn{2}{|l|}{ Reading simulated signal } \\
\hline Input port used to graph the simulated signal & Connector0/AI2 \\
\hline
\end{tabular}

Note: All electrical connections within the FPGA are here provided. By wiring these connectors adequately, one can run the LabVIEW FPGA programs described in Figures S2S5. FPGA, field programmable gate array; PMT, photomultiplier tube. 
colleagues [24].

\section{Passive droplet merger for the addition of other reagents}

The third droplet microfluidic function, reviewed here, is a passive droplet merger developed by Hosokawa and colleagues [12]. This function enables the addition of precise amounts of reagents to each droplet for further processing. For example, droplet merging has been used to add multiple displacement amplification (MDA) reagents to cell lysate droplets to amplify single-cell genomes [12]. Likewise, others have merged droplets with barcoding DNA sequences [66] to tag and then identify each experiment. In general, droplet merging is a useful tool to enable multiplestep reactions in droplet microfluidics at high throughput.

There are active $[7,65,74-77]$ and passive $[12,14,78-80]$ droplet merging devices. Regardless of the merging approach, 1:1 droplet synchronization is one of the most challenging aspects. Our group has published the experimental details of the device described in this section [12]. When this system runs efficiently, it can yield a droplet merging success rate of $95 \%$ at a rate as high as 12,000 droplets/min. In this merging device, partially stabilized droplets are merged with newly formed droplets of a particular reagent (e.g., lysis buffer, MDA reagents, and barcoding). Once the droplets are merged at the zigzag channel, an additional surfactant is added to stabilize the newly formed droplets.

Droplet merging often implies reinjecting already-made droplets into a merging device. If one is not careful with this reinjection process, droplets can be poorly packed together and unevenly spaced, leading to synchronization problems when merging in a 1:1 ratio.

For a successful reinjection, the droplet suspension must settle first until most of the droplets aggregate at the top of the container. Then these closely packed droplets can be carefully retrieved using a piece of tubing and a syringe pump with a constant withdrawing rate (e.g., $10 \mu \mathrm{l} / \mathrm{min}$ ). Once the tubing is loaded with the droplets, one can detach the tubing with the droplet suspension from the syringe pump and connect it directly to the merging chip.

\section{Results and discussion}

In this section, we discuss the overall integration of the three microfluidic techniques presented here and show a practical way for achieving the high-throughput requirements of single-cell functional metagenomics studies.

In functional metagenomics $[81,82]$, characterization of metagenomic fragments is essential for finding applicable values as genetic resources. In practice, functional metagenomics deals with several experimental processes, such as extraction of microbial DNA from environmental samples, construction of an expression library with the microbial genetic material, and a screening method for detecting the desired phenotypic responses. The first two processes are obtained in large quantities (e.g., millions of samples at a time), and they are discussed elsewhere [83]. However, the third process is more challenging because it involves searching for different phenotypes in each cell in a library of millions. Therefore, traditional techniques in plates cannot practically handle the number of experiments required. The required screening throughput can only be achieved using droplet-based microfluidic methods. For these reasons, in this study, we decided to integrate the three droplet microfluidic techniques to enable a practical screening of functional metagenomic libraries $[6,12]$ in a single platform. This integration can overcome the obstacles that were not adequately addressed previously.

For example, the protocol by Mazutis et al. [6] lacks the capability of adding precise amounts of regents to alreadyformed droplets as these droplets are closed micro-reaction chambers with no extra inputs. Similarly, the protocol by Hosokawa et al. [12] massively overcomes this limitation with a droplet merger, but unfortunately, their throughput is tremendously hindered by manually sorting the droplets with a pipette.

Our combinatory approach of the three droplet microfluidic tools is described in Figure $\mathbf{5}$ and in Movie S1. The output of the first technology is the encapsulation of millions of single cells in microdroplets (Figure 5A). This process is carried out at a cell concentration of 0.1 cells/droplet, and our device generates droplets at 250-350 droplets/s. Therefore, a hundred thousand cells can be encapsulated in approximately $1 \mathrm{~h}$.

With the second technology, one can automatically sort out the droplets that show positive fluorescence (i.e., functional metagenomic information) from the negative ones (Figure 5B). Our sorting system offers a quick electrical response from fluorescence detection to the triggering signal, as only $360 \mu$ s is needed per event. However, the limiting factor in the sorting process is the speed at which the droplets can move through the detection zone without compromising their integrity. Therefore, our automatic droplet sorting device achieved a maximum sorting rate of 60 droplet/s. Sorting half of the droplet population produced (i.e., $\sim 500,000$ droplets or $\sim 50,000$ droplets with single cells) takes roughly $2.5 \mathrm{~h}$.

Finally, the third chip enables us to combine those positive-fluorescence droplets with other reagents for further processing or amplification (Figure 5C). The droplet merging device shows a success rate of $95 \%$ at a high throughput of 200 droplets/s. Therefore, merging the positive-fluorescence droplets (e.g., sorted out from the previous device) with lysis buffer or amplification reagents can 


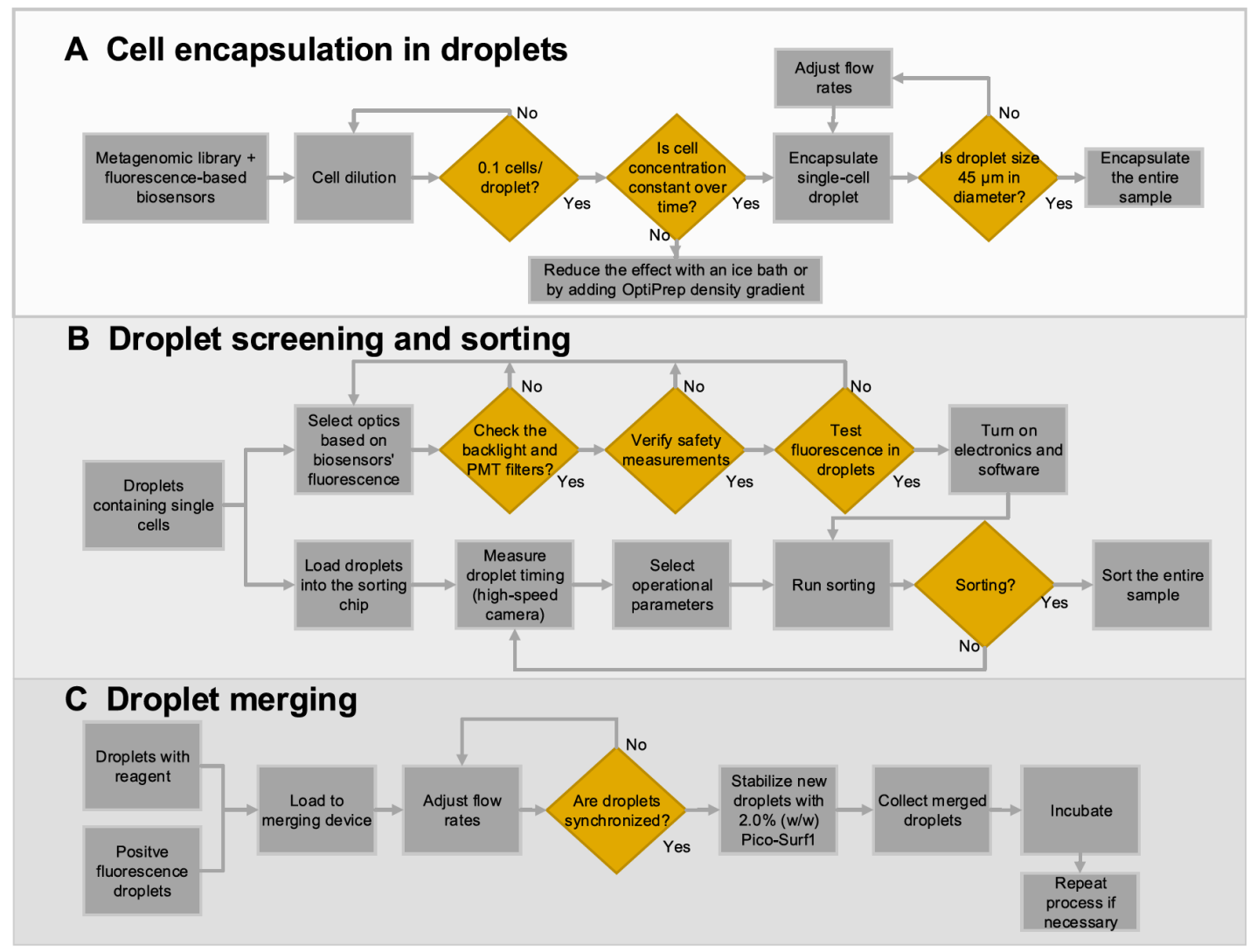

Figure 5 Droplet microfluidic capabilities enabling functional metagenomic studies

This figure summarizes the three droplet microfluidic tools integrated in this study. A. Process diagram fully describing single-cell encapsulation. A critical step of this procedure is to measure the cell concentration before the encapsulation to guarantee that only one cell is encapsulated per droplet. B. Protocol for droplet sorting. This protocol includes selecting the right optical components and identifying possible errors on the hardware and software. Measuring the time from droplet detection to the sorting junction is fundamental for synchronizing the control system with the device's droplet flow. C. Merging process of two droplets to add new reagents to already-made droplets. The most crucial step in this process is to adjust the inlet flow rates to obtain a 1:1 droplet synchronization.

take less than $0.5 \mathrm{~h}$.

Once the metagenomic material is obtained from the positive droplets, the next steps consist of performing sequencing and bioinformatics.

This protocol allows the finding of functional genes out of vast metagenomic libraries. Our group is currently working on a case study that focuses on the metagenomic application of the methods presented in this publication. In this follow-up work, we apply the techniques presented here to search for lipolytic enzymes in Red Sea environmental samples. This metagenomic investigation lead to identifying three novel lipase genes. These genes showed thermostability and high activity based on alignment tests and functional assay results.

\section{Conclusion}

A recipe for bulk micromachining high-quality and longlasting master molds on silicon is provided for the first time.
Although our procedure requires specialized DRIE equipment, it shows a more robust solution to mold manufacturing than traditional master mold techniques such as SU-8 lithography because these molds can be used indefinitely (e.g., $>100$ times) without showing signs of wear.

Three microfluidic droplet techniques for single-cell studies are also described: single-cell encapsulation, fluorescence-based automatic droplet sorting, and droplet merging. For encapsulation, cell concentration is the most crucial parameter, and therefore it must be measured before starting any experiment. A concentration of 0.1 cells/droplet provides the best results to obtain single-cell encapsulation. Our droplet encapsulation system generates droplets at rates of $\sim 250-350$ droplets/s and effectively encapsulates approximately 25 droplets/s. Automatic droplet sorting is then described entirely, providing guides to optics, instrumentation, and controls. The electronics response time from fluorescence detection to the activation of triggering signal takes only $360 \mu$ s per event. However, controlled delays are programmed as we can only move the droplets 
at $\sim 60$ droplets/s through the channel without tearing them apart. Our setup is robust and can work with four wavelengths of fluorescent excitation (i.e., 405, 488, 561, and $637 \mathrm{~nm}$ ), making it compatible with several fluorescenceemitting biosensors. A summary of fluorochromes compatible with this setup is also provided as a quick selection guide. We present a passive droplet merging device that can achieve efficiencies of $95 \%$ and at $\sim 200$ droplets/s. This merger facilitates the addition of precise amounts of reagents for various purposes, such as starting or terminating a reaction and adding new reagents.

Finally, we discuss the potential of these three microfluidic technologies in mining the immense pool of metagenomic resources for bioprospecting.

\section{CRediT author statement}

David Conchouso: Methodology, Investigation, Writing original draft, Writing - review \& editing. Amani Al-Ma'abadi: Resources. Hayedeh Behzad: Resources. Mohammed Alarawi: Resources. Masahito Hosokawa: Methodology. Yohei Nishikawa: Methodology. Haruko Takeyama: Methodology. Katsuhiko Mineta: Methodology, Supervision, Writing - review \& editing. Takashi Gojobori: Conceptualization, Methodology, Supervision, Writing - review \& editing. All authors have read and approved the final manuscript.

\section{Competing interests}

The authors have declared no competing interests.

\section{Acknowledgments}

The work was supported by the grants from King Abdullah University of Science and Technology (KAUST), Saudi Arabia (Grant Nos. BAS/1/1059/01/01, URF/1/1976/03/01, URF/1/1976-17-01, URF/1/1976-20-01, and FCS/1/332601-01). Finally, we thank the nanofabrication and the microfluidics Core Laboratories at KAUST for their support in realizing these microfluidic technologies.

\section{Supplementary material}

Supplementary data to this article can be found online at https://doi.org/10.1016/j.gpb.2021.12.018.

\section{ORCID}

0000-0002-9788-0977 (David Conchouso) 0000-0001-8895-5423 (Amani Al-Ma'abadi) 0000-0001-7216-8337 (Hayedeh Behzad)
0000-0001-8986-8209 (Mohammed Alarawi)

0000-0002-7070-4690 (Masahito Hosokawa)

0000-0001-5443-7230 (Yohei Nishikawa)

0000-0002-2058-8185 (Haruko Takeyama)

0000-0002-4727-045X (Katsuhiko Mineta)

0000-0001-7850-1743 (Takashi Gojobori)

\section{References}

[1] Song H, Chen DL, Ismagilov RF. Reactions in droplets in microfluidic channels. Angew Chem Int Ed 2006;45:7336-56.

[2] DeMello AJ. Control and detection of chemical reactions in microfluidic systems. Nature 2006;442:394-402.

[3] Whitesides GM. The origins and the future of microfluidics. Nature 2006;442:368-73.

[4] Shui L, Eijkel JCT, van den Berg A. Multiphase flow in microfluidic systems - control and applications of droplets and interfaces. Adv Colloid Interface Sci 2007;133:35-49.

[5] Teh SY, Lin R, Hung LH, Lee AP. Droplet microfluidics. Lab Chip 2008;8:198-220.

[6] Mazutis L, Gilbert J, Ung WL, Weitz DA, Griffiths AD, Heyman JA. Single-cell analysis and sorting using droplet-based microfluidics. Nat Protoc 2013;8:870-91.

[7] Lan F, Demaree B, Ahmed N, Abate AR. Single-cell genome sequencing at ultra-high-throughput with microfluidic droplet barcoding. Nat Biotechnol 2017;35:640-6.

[8] Zilionis R, Nainys J, Veres A, Savova V, Zemmour D, Klein AM, et al. Single-cell barcoding and sequencing using droplet microfluidics. Nat Protoc 2017;12:44-73.

[9] Gawad C, Koh W, Quake SR. Single-cell genome sequencing: current state of the science. Nat Rev Genet 2016;17:175-88.

[10] Hosokawa M, Nishikawa Y, Kogawa M, Takeyama H. Analysis of environmental bacteria at single-cell level. International Conference on Solid-state Sensors 2017:634-7.

[11] Hosokawa M, Hoshino Y, Nishikawa Y, Hirose T, Yoon DH, Mori T, et al. Droplet-based microfluidics for high-throughput screening of a metagenomic library for isolation of microbial enzymes. Biosens Bioelectron 2015;67:379-85.

[12] Hosokawa M, Nishikawa Y, Kogawa M, Takeyama H. Massively parallel whole genome amplification for single-cell sequencing using droplet microfluidics. Sci Rep 2017;7:5199.

[13] Brouzes E, Medkova M, Savenelli N, Marran D, Twardowski M, Hutchison JB, et al. Droplet microfluidic technology for single-cell high-throughput screening. Proc Natl Acad Sci U S A 2009;106:14195-200.

[14] Niu X, Gulati S, Edel JB, deMello AJ. Pillar-induced droplet merging in microfluidic circuits. Lab Chip 2008;8:1837.

[15] Ma S, Murphy TW, Lu C. Microfluidics for genome-wide studies involving next generation sequencing. Biomicrofluidics 2017;11:021501.

[16] Klein AM, Mazutis L, Akartuna I, Tallapragada N, Veres A, Li V, et al. Droplet barcoding for single-cell transcriptomics applied to embryonic stem cells. Cell 2015;161:1187-201.

[17] Macosko EZ, Basu A, Satija R, Nemesh J, Shekhar K, Goldman $\mathrm{M}$, et al. Highly parallel genome-wide expression profiling of individual cells using nanoliter droplets. Cell 2015;161:1202-14.

[18] Lagus TP, Edd JF. A review of the theory, methods and recent applications of high-throughput single-cell droplet microfluidics. J Phys D Appl Phys 2013;46:114005.

[19] Castro D, Conchouso D, Kodzius R, Arevalo A, Foulds IG. Highthroughput incubation and quantification of agglutination assays in a microfluidic system. Genes 2018;9:281.

[20] Conchouso D, McKerricher G, Arevalo A, Castro D, Shamim A, Foulds IG. Comparison of capacitive and radio frequency resonator 
sensors for monitoring parallelized droplet microfluidic production. Lab Chip 2016;16:3210-9.

[21] Schaerli Y, Wootton RC, Robinson T, Stein V, Dunsby C, Neil MAA, et al. Continuous-flow polymerase chain reaction of singlecopy DNA in microfluidic microdroplets. Anal Chem 2009;81:302-6.

[22] Yin H, Marshall D. Microfluidics for single cell analysis. Curr Opin Biotechnol 2012;23:110-9.

[23] Lecault V, White AK, Singhal A, Hansen CL. Microfluidic single cell analysis: from promise to practice. Curr Opin Chem Biol 2012;16:381-90.

[24] Basu AS. Droplet morphometry and velocimetry (DMV): a video processing software for time-resolved, label-free tracking of droplet parameters. Lab Chip 2013;13:1892-901.

[25] Erickson D, O'Dell D, Jiang L, Oncescu V, Gumus A, Lee S, et al. Smartphone technology can be transformative to the deployment of lab-on-chip diagnostics. Lab Chip 2014;14:3159-64.

[26] Rinke C, Lee J, Nath N, Goudeau D, Thompson B, Poulton N, et al. Obtaining genomes from uncultivated environmental microorganisms using FACS-based single-cell genomics. Nat Protoc 2014;9:1038-48.

[27] Kogawa M, Hosokawa M, Nishikawa Y, Mori K, Takeyama H. Obtaining high-quality draft genomes from uncultured microbes by cleaning and co-assembly of single-cell amplified genomes. Sci Rep 2018;8:577.

[28] Kodzius R, Gojobori T. Marine metagenomics as a source for bioprospecting. Mar Genomics 2015;24:21-30.

[29] Sleator RD, Shortall C, Hill C. Metagenomics. Lett Appl MicroBiol 2008;47:361-6.

[30] Kodzius R, Gojobori T. Single-cell technologies in environmental omics. Gene 2016;576:701-7.

[31] Amann RI, Ludwig W, Schleifer KH. Phylogenetic identification and in situ detection of individual microbial cells without cultivation. Microbiol Rev 1995;59:143-69.

[32] Conchouso D, Castro D, Khan SA, Foulds IG. Three-dimensional parallelization of microfluidic droplet generators for a litre per hour volume production of single emulsions. Lab Chip 2014;14:301120.

[33] Mckerricher G, Conchouso D, Cook BS, Foulds IG, Shamim A. Crude oil water-cut sensing with disposable laser ablated and inkjet printed RF microfluidics. IEEE MTT-S International Microwave Symposium 2014:1-3.

[34] Mineta K, Gojobori T. Databases of the marine metagenomics. Gene 2016;576:724-8.

[35] Alma'abadi AD, Gojobori T, Mineta K. Marine metagenome as a resource for novel enzymes. Genomics Proteomics Bioinformatics 2015;13:290-5.

[36] Gojobori T, Chiang TY. Opening a new era of "ecological genetics and genomics". Ecol Genet Genom 2016;1:8.

[37] Behzad H, Ibarra MA, Mineta K, Gojobori T. Metagenomic studies of the Red Sea. Gene 2016;576:717-23.

[38] Li H, Fan Y, Conchouso D, Foulds IG. $\mathrm{CO}_{2}$ laser-induced bump formation and growth on polystyrene for multi-depth soft lithography molds. J Micromech Microeng 2012;22:115037.

[39] Fan Y, Li H, Yi Y, Foulds IG. Laser micromachined wax-covered plastic paper as both sputter deposition shadow masks and deepultraviolet patterning masks for polymethylmethacrylate-based microfluidic systems. J Micro-Nanolith MEM 2013;12:049701.

[40] Kricka LJ, Fortina P, Panaro NJ, Wilding P, Alonso-Amigo G, Becker H. Fabrication of plastic microchips by hot embossing. Lab Chip 2002;2:1.

[41] Attia UM, Marson S, Alcock JR. Micro-injection moulding of polymer microfluidic devices. Microfluid Nanofluidics 2009;7:128.

[42] Buttner U, Sivashankar S, Agambayev S, Mashraei Y, Salama $\mathrm{KN}$. Flash $\mu$-fluidics: a rapid prototyping method for fabricating microfluidic devices. RSC Adv 2016;6:74822-32.

[43] Bhattacharjee N, Urrios A, Kang S, Folch A. The upcoming 3Dprinting revolution in microfluidics. Lab Chip 2016;16:1720-42.

[44] Au AK, Huynh W, Horowitz LF, Folch A. 3D-printed microfluidics. Angew Chem Int Ed Engl 2016;55:3862-81.

[45] Li EQ, Zhang JM, Thoroddsen ST. Simple and inexpensive microfluidic devices for the generation of monodisperse multiple emulsions. J Micromech Microeng 2014;24:015019.

[46] Castro D, Ingram P, Kodzius R, Conchouso D, Yoon E, Foulds IG. Characterization of solid UV cross-linked PEGDA for biological applications. Proc IEEE Int Conf Micro Electro Mech Syst 2013,26:457-60.

[47] Kim P, Kwon KW, Park MC, Lee SH, Kim SM, Suh KY. Soft lithography for microfluidics: a review. Biochip J 2008;2:1-11.

[48] Xia Y, Whitesides GM. Soft lithography. Angew Chem Int Ed Engl 1998;37:550-75.

[49] Zhou J, Khodakov DA, Ellis AV, Voelcker NH. Surface modification for PDMS-based microfluidic devices. Electrophoresis 2012;33:89-104.

[50] Cho W, Ko YJ, Ahn YM, Yoon JY, Cho NG. Surface modification effect of wettability on the performance of PDMS-based valveless micropump. Key Eng Mater 2006;326-328:297-300.

[51] Vlachopoulou ME, Petrou PS, Kakabakos SE, Tserepi A, Beltsios K, Gogolides E. Effect of surface nanostructuring of PDMS on wetting properties, hydrophobic recovery and protein adsorption. Microelectron Eng 2009;86:1321-4.

[52] Xia Y, Qin D, Yin Y. Surface patterning and its application in wetting/dewetting studies. Curr Opin Colloid Interface Sci 2001;6:54-64.

[53] Castro D, Conchouso D, Fan Y, Foulds IG. Surface treatments of soft molds for high aspect ratio molding of Poly-PEGDA. Proceedings of the 16th International Conference on Miniaturized Systems for Chemistry and Life Sciences, MicroTAS 2012:1231-3.

[54] Lin CH, Lee GB, Chang BW, Chang GL. A new fabrication process for ultra-thick microfluidic microstructures utilizing SU-8 photoresist. J Micromech Microeng 2002;12:590-7.

[55] Zhang XL, Du LQ, Zhu SM. Investigation on internal stress of SU8 photoresist film. Key Eng Mater 2014;613:251-8.

[56] Park HW, Kim HJ, Roh JH, Choi JK, Cha KR. Simple and costeffective method for edge bead removal by using a taping method. J Korean Phys Soc 2018;73:1473-8.

[57] Gao JX, Yeo LP, Chan-Park MB, Miao JM, Yan YH, Sun JB, et al. Antistick postpassivation of high-aspect ratio silicon molds fabricated by deep-reactive ion etching. J Microelectromech Syst 2006;15:84-93.

[58] Fu YQ, Colli A, Fasoli A, Luo JK, Flewitt AJ, Ferrari AC, et al. Deep reactive ion etching as a tool for nanostructure fabrication. $J$ Vac Sci Technol B Microelectron Nanometer Struct Process Meas Phenom 2009;27:1520.

[59] Jang EJ, Park YB, Lee HJ, Choi DG, Jeong JH, Lee ES, et al. Effect of surface treatments on interfacial adhesion energy between UV-curable resist and glass wafer. Int J Adhes Adhes 2009;29:6629.

[60] Colin PY, Kintses B, Gielen F, Miton CM, Fischer G, Mohamed $\mathrm{MF}$, et al. Ultrahigh-throughput discovery of promiscuous enzymes by picodroplet functional metagenomics. Nat Commun 2015;6:10008.

[61] Kintses B, Hein C, Mohamed MF, Fischlechner M, Courtois F, Lainé $\mathrm{C}$, et al. Picoliter cell lysate assays in microfluidic droplet compartments for directed enzyme evolution. Chem Biol 2012;19:1001-9.

[62] Agresti JJ, Antipov E, Abate AR, Ahn K, Rowat AC, Baret JC, et al. Ultrahigh-throughput screening in drop-based microfluidics for directed evolution. Proc Natl Acad Sci U S A 2010;107:4004-9.

[63] Köster S, Angilè FE, Duan H, Agresti JJ, Wintner A, Schmitz C, et al. Drop-based microfluidic devices for encapsulation of single 
cells. Lab Chip 2008;8:1110.

[64] Anna SL, Bontoux N, Stone HA. Formation of dispersions using "flow focusing" in microchannels. Appl Phys Lett 2003;82:364-6.

[65] Lan F, Haliburton JR, Yuan A, Abate AR. Droplet barcoding for massively parallel single-molecule deep sequencing. Nat Commun 2016;7:11784.

[66] Liu X, Painter RE, Enesa K, Holmes D, Whyte G, Garlisi CG, et al. High-throughput screening of antibiotic-resistant bacteria in picodroplets. Lab Chip 2016;16:1636-43.

[67] Wang BL, Ghaderi A, Zhou H, Agresti J, Weitz DA, Fink GR, et al. Microfluidic high-throughput culturing of single cells for selection based on extracellular metabolite production or consumption. Nat Biotechnol 2014;32:473-8.

[68] Zheng H, Ho PY, Jiang M, Tang B, Liu W, Li D, et al. Interrogating the Escherichia coli cell cycle by cell dimension perturbations. Proc Natl Acad Sci U S A 2016;113:15000-5.

[69] Sciambi A, Abate AR. Accurate microfluidic sorting of droplets at $30 \mathrm{kHz}$. Lab Chip 2014;15:47-51.

[70] Baret JC, Miller OJ, Taly V, Ryckelynck M, El-Harrak A, Frenz L, et al. Fluorescence-activated droplet sorting (FADS): efficient microfluidic cell sorting based on enzymatic activity. Lab Chip 2009;9:1850-8.

[71] Xi HD, Zheng H, Guo W, Gañán-Calvo AM, Ai Y, Tsao CW, et al. Active droplet sorting in microfluidics: a review. Lab Chip 2017;17:751-71.

[72] Ahn K, Kerbage C, Hunt TP, Westervelt RM, Link DR, Weitz DA. Dielectrophoretic manipulation of drops for high-speed microfluidic sorting devices. Appl Phys Lett 2006;88:024104.

[73] Antonio-Torres D, Villanueva-Perez D, Sanchez-Canepa E, Segura-Meraz N, Garcia-Garcia D, Conchouso D, et al. A PicoBlazebased embedded system for monitoring applications. International Conference on Electrical Communications and Computers
2009:173-7.

[74] Zagnoni M, Baroud CN, Cooper JM. Electrically initiated upstream coalescence cascade of droplets in a microfluidic flow. Phys Rev E 2009;80:046303.

[75] Varma VB, Ray A, Wang ZM, Wang ZP, Ramanujan RV. Droplet merging on a Lab-on-a-Chip platform by uniform magnetic fields. Sci Rep 2016;6:37671.

[76] Xu B, Nguyen NT, Neng Wong T. Droplet coalescence in microfluidic systems. Micro and Nanosystems 2011;3:131-6.

[77] Jamshaid A, Igaki M, Yoon DH, Sekiguchi T, Shoji S. Controllable active microdroplets merging device using horizontal pneumatic microvalves. Micromachines 2013;4:34-48.

[78] Christopher GF, Bergstein J, End NB, Poon M, Nguyen C, Anna SL. Coalescence and splitting of confined droplets at microfluidic junctions. Lab Chip 2009;9:1102-9.

[79] Fidalgo LM, Abell C, Huck WTS. Surface-induced droplet fusion in microfluidic devices. Lab Chip 2007;7:984.

[80] Korczyk PM, Derzsi L, Jakieła S, Garstecki P. Microfluidic traps for hard-wired operations on droplets. Lab Chip 2013;13:4096102.

[81] Johnson J, Jain K, Madamwar D. 2-functional metagenomics: exploring nature's gold mine. In: Gunasekaran P, Noronha S, Pandey A, editors. Current developments in biotechnology and bioengineering. Amsterdam: Elsevier B.V.; 2017, p.27-43.

[82] Lam KN, Cheng J, Engel K, Neufeld JD, Charles TC. Current and future resources for functional metagenomics. Front Microbiol 2015;6:149

[83] Stein JL, Marsh TL, Wu KY, Shizuya H, DeLong EF. Characterization of uncultivated prokaryotes: isolation and analysis of a 40kilobase-pair genome fragment from a planktonic marine archaeon. J Bacteriol 1996;178:591-9. 